\title{
A new bacmid for customized protein glycosylation pathway engineering in the baculovirus-insect cell system
}

Ajay B. Maghodia ${ }^{a, \#, 1}$, Christoph Geisler ${ }^{a, \#, 2}$, and Donald L. Jarvis ${ }^{a, b,{ }^{*}}$

${ }^{a}$ GlycoBac, LLC, Laramie, WY 82072

${ }^{\mathrm{b}}$ Department of Molecular Biology, University of Wyoming, Laramie, WY 82071

${ }^{1}$ Present Address: Sanofi US, 225 2nd Avenue, Waltham MA 02451, USA.

${ }^{2}$ Present Address: Theraneutrics, 1938 Harney Street, Laramie, WY 82072, USA.

"Corresponding author. Department of Molecular Biology, University of Wyoming, Laramie, WY, USA. Tel.: +1-307-766-4282. E-mail address: dljarvis@uwyo.edu 
Table of Contents

1. Supplementary Methods.

2. Supplementary Table S1.

3. Supplementary Figure Legends.

4. Supplementary Fig. S1.

5. Supplementary Fig. S2.

6. Supplementary Fig. S3.

7. Supplementary Fig. S4.

8. Supplementary Fig. S5.

9. Supplementary Fig. S6. 


\section{Supplementary Methods}

\section{General DNA manipulations}

All primers were from Integrated DNA Technologies (Coralville, lowa) and their sequences are given in Supplementary Table S1. PCR reactions were performed using Phusion DNA polymerase (New England Biolabs, Ipswich, MA) in HF buffer provided by the manufacturer. PCR products and DNA fragments obtained by restriction digests were purified for further analysis by agarose gel electrophoresis using the E.Z.N.A. Gel Extraction Kit (Omega Bio-tek, Norcross, GA). Restriction digests were carried out using restriction enzymes from New England Biolabs in the manufacturer's recommended reaction buffers. DNA ligations were performed using the Quick Ligation kit (New England Biolabs).

Plasmid and bacmid identities were confirmed after each manipulation by restriction mapping and all PCR amplimer inserts were verified by sequencing (Genewiz, South Plainfield, NJ).

All bacterial transformations were accomplished by electroporation using a Bio-Rad Gene Pulser with $2 \mathrm{~mm}$ gap electroporation cuvettes. Electrocompetent cells were prepared as described previously ${ }^{1}$. Selection plates included the indicated concentrations of the following antibiotics: kanamycin $(50 \mu \mathrm{g} / \mathrm{mL})$, ampicillin $(100$ $\mu \mathrm{g} / \mathrm{mL})$, gentamycin $(7 \mu \mathrm{g} / \mathrm{mL})$, chloramphenicol (35 $\mu \mathrm{g} / \mathrm{mL})$, X-Gal $(100 \mu \mathrm{g} / \mathrm{mL})$, IPTG $(40 \mu \mathrm{g} / \mathrm{mL})$, zeocin $(10 \mu \mathrm{g} / \mathrm{mL})$.

\section{PolyBac construction}

Tn7 transposition site (baculovirus polh locus).

A plasmid containing a synthetic DNA encoding two multiple cloning sites (MCS -L and $-\mathrm{R}$ ), a kanamycin resistance marker, and a Lac-promoter-driven E. coli LacZa fragment fused in-frame to a 5' mini-attTn7 insertion sequence ('LacZa-mini-attTn7') was purchased from DNA2.0 (ATUM, Newark, CA; Supplementary Figure 1A).

Sequences flanking the AcMNPV polh coding sequence, a mini-F replicon, and the AcMNPV hr2 enhancer were then cloned into this plasmid in three steps. First, sequences flanking the AcMNPV polh coding sequence were PCR amplified with PolhUS and PolhDS primer pairs (Supplementary Table 1), digested with Pvul/EcoRV and Fsel/Mlul, respectively, and sequentially inserted into the Pacl/Pmel (Supplementary Figure 1A-B) and Fsel/Ascl (Supplementary Figure 1B-C) sites of the plasmid containing the synthetic DNA. The resulting plasmid was designated pUC.LacZa-mini-attTn7.polh and contained $1367 \mathrm{bp}$ of the sequence immediately upstream and $1756 \mathrm{bp}$ of the sequence immediately downstream of the polh ORF (Supplementary Figure 1C). These regions were inserted to enable site-specific recombination of the LacZa-mini-attTn7 element into the AcMNPV polh locus with concomitant deletion of the polh gene.

Second, the mini-F replicon was excised from pBeloBAC11 (New England Biolabs) using Nrul and inserted into the pUC.LacZa-mini-attTn7.polh Avrll site, yielding pUC.LacZa-mini-attTn7.mini-F.polh (Supplementary Figure 1C-D). The mini-F replicon 
allows for single-copy replication of very large DNA fragments in bacterial cells and does not interfere with baculovirus replication in insect cells ${ }^{2,3}$.

Third, the AcMNPV hr2 enhancer element was amplified with the HR2SP and ASP primer pair, digested with $S p h l$ and BstBI, and inserted into these same sites in pUC.LacZa-mini-attTn7.mini-F.polh. The resulting transfer plasmid was designated pPBTV-1 (Supplementary Figure 1E) and the annotated pPBTV-1 sequence file is included in Supplementary Materials (pPBTV-1.gb).

Finally, pPBTV-1 and AcMNPV strain E2 genomic DNA 4 were mixed and used for homologous recombination in Sf-rhabdovirus-negative (Sf-RVN) Sf cells ${ }^{5}$ as described previously ${ }^{6}$. The resulting baculovirus progeny were harvested, resolved by plaque assay, and recombinant viral plaques were tentatively identified by the occlusionnegative plaque phenotype resulting from deletion of the polh gene. Further confirmation was obtained by using PCR to amplify sequences spanning the expected recombination junctions using primer pairs internal and external to the transfer plasmid, then sequencing the amplimers. A confirmed recombinant virus was amplified and used to isolate genomic viral DNA, which was then transformed into E. coli $\mathrm{DH} 10 \mathrm{~B}^{7}$ (New England Biolabs). The transformed cells were selected with kanamycin, X-Gal, and IPTG, and bacmid DNA was isolated from kanamycin resistant blue $E$. coli clones, validated by restriction mapping, and designated $\Delta$ polh-bacmid.

Gateway $^{\mathrm{TM}}$ and Cre-LoxP recombination sites (baculovirus egt locus)

A plasmid containing a synthetic DNA sequence encoding an AcMNPV p10promoter-driven LacZ-Rudolph Red Fluorescent Protein (RFP) fusion protein, an AcMNPV ie1-promoter-driven herpes simplex virus type 2 thymidine kinase (TK) gene, Gateway $^{\mathrm{TM}}$ (attR1/R2) recombination sites, a LoxP recombination site, and two multiple cloning sites (MCS $-L$ and $-R$ ), was purchased from DNA2.0 (Supplementary Figure 2A).

Sequences flanking the AcMNPV egt coding sequence were then amplified with EGTUS and EGTDS primer pairs and the resulting upstream and downstream flanking sequences were digested with EcoRVIAgel and Nhel/Fsel, respectively. The digested $\mathrm{PCR}$ products were then sequentially inserted into the Pmel/Agel (Supplementary Figure 2B) and Avrll/Fsel (Supplementary Figure 2C) sites of the plasmid carrying the synthetic DNA. The resulting plasmid was designated pPBTV-2 and included $2175 \mathrm{bp}$ of the sequence immediately upstream and $2123 \mathrm{bp}$ of the sequence immediately downstream of the egt ORF (Supplementary Figure 2C). These regions were inserted to enable site-specific recombination of the Cre-LoxP and Gateway ${ }^{\mathrm{TM}}$ (attR1/R2) recombination sites and selectable markers into the AcMNPV egt locus with concomitant deletion of the egt gene. The annotated pPBTV-2 sequence file is included in Supplementary Materials (pPBTV-2.gb).

pPBTV-2 was subsequently mixed with Avrll-linearized $\Delta$ polh-bacmid and the mixture was used to co-transfect Sf-RVN cells for homologous recombination. The baculovirus progeny were harvested and resolved by plaque assay. Recombinant viral plaques were tentatively identified by their blue and red plaque phenotypes in the presence of X-gal resulting from expression of the LacZ-RFP fusion protein. The 
genotype of a putative recombinant was confirmed by using PCR to amplify the expected recombination junctions with primers internal and external to the transfer plasmid and sequencing the resulting amplimers. A validated recombinant virus was then amplified and genomic viral DNA was isolated, used to transform E. coli $\mathrm{DH} 10 \mathrm{~B}^{7}$, and transformants were selected on agar plates containing kanamycin, X-Gal, and IPTG. Bacmid DNAs isolated from kanamycin resistant blue E. coli clones were analyzed by restriction mapping and a validated clone was designated $\Delta$ polh- $\Delta$ egtbacmid.

\section{ChiA and v-cath deletions}

We subsequently deleted the AcMNPV chiA and $v$-cath genes from $\Delta$ polh- $\Delta$ egtbacmid using the $\lambda$-red recombination system to isolate the final, parental bacmid used in this study, which was designated PolyBac. This was accomplished as follows.

First, we transformed E. coli BW25113 harboring the pKD46 helper plasmid encoding $\lambda$-red recombinase (Coli Genetic Stock Center, Yale University, New Haven, CT) with $\Delta$ polh- $\Delta$ egt-bacmid and selected the transformed cells on agar plates containing kanamycin and ampicillin.

Second, we designed a synthetic DNA encoding the $S$. hindustanus bleomycin gene conferring zeocin resistance flanked by $50 \mathrm{bp}$ of DNA derived from the AcMNPV chiA and $v$-cath genes. We then purchased this synthetic DNA from Integrated DNA Technologies and used the plasmid carrying this DNA to transform the E. coli BW25113 strain harboring the pKD46 helper plasmid and $\Delta p o l h-\Delta e g t$-bacmid. Transformants were selected on agar plates containing kanamycin and zeocin and confirmed the zeocin resistance cassette was correctly integrated at the chiA/v-cath locus by restriction mapping.

Finally, we confirmed the presence and sequences of all inserts in this construct by sequencing. This validated bacmid was designated PolyBac and PolyBac DNA was isolated and used to transform E. coli DH10B (Figure 2A). The annotated PolyBac sequence file is included in Supplementary Materials (PolyBac.gb).

Construction of Tn7 helper and isolation of E.coli PolyBac ${ }^{\mathrm{DH} 10 \mathrm{~B}}$

$\mathrm{Tn} 7$ helper is a plasmid that includes a synthetic DNA encoding the $\mathrm{Tn} 7$ transposase genes tnsA-D under control of the IPTG-inducible LacZ promoter, as well as an ampicillin resistance marker. This DNA was designed and purchased from DNA2.0. Its genetic map is shown in Supplementary Figure 3 and the annotated Tn7 helper sequence file is included in Supplementary Materials (Tn7 helper.gb). This Tn7 helper plasmid was transformed into the E. coli DH10B strain harboring PolyBac, transformants were selected on agar plates containing kanamycin, zeocin, and ampicillin, and a triple resistant clone was designated PolyBac ${ }^{\mathrm{DH} 10 \mathrm{~B}}$ (Figure 2A).

\section{Transfer vector constructions}

Construction of Tn7 TV1

A dual-purpose transfer vector designed to transfer multiple genes into the miniattTn7 or attR1/R2 sites in PolyBac was designed and purchased from GeneArt. This 
plasmid included $\operatorname{Tn} 7$ ( $\mathrm{Tn} 7 \mathrm{R}$ and $\mathrm{Tn} 7 \mathrm{~L}$ ) recognition sites, Gateway ${ }^{\mathrm{TM}}$ recombination (attL1 and attL2) sites, a gentamycin resistance marker, two multiple cloning sites (MCS $-\mathrm{L}$ and $-\mathrm{R}$ ), and an AcMNPV p6.9-promoter-driven expression cassette encoding $\mathrm{rH} 5$. The rH5 open reading frame encoded an 8X-HIS-tagged influenza A/Vietnam/1203/04 (H5N1) $\mathrm{HA}_{0}$ ectodomain with its furin cleavage site deleted, an AcMNPV egt signal sequence, and a C-terminal trimerization domain. These features are highlighted in the genetic map at the top of Supplementary Figure 4, above the Tn7 TV1 genetic map, and are similar to those of a previously described $\mathrm{rH} 5$ construct $^{8}$. The plasmid purchased from GeneArt had a kanamycin resistance marker, as does PolyBac, which would have interfered with a downstream antibiotic selection step. Therefore, the entire synthetic DNA cassette was excised using flanking $\mathrm{BamHI}$ sites and subcloned into the $\mathrm{BamHI}$ site of pUC19, which has an ampicillin resistance marker, and the resulting transfer plasmid was designated Tn7 TV1 (Supplementary Figure 4A). This vector can be used to insert genes into the PolyBac attR1/R2 sites or mini-attTn7 site and the rH5 open reading frame can be easily replaced by another gene of interest. The annotated Tn7 TV1 sequence file is included in Supplementary Materials (Tn7 TV1.gb).

\section{Construction of Tn7 TV3}

Because we only planned to use the Tn7 sites of Tn7 TV1 to create the downstream constructs needed for this project, we deleted the Gateway ${ }^{\mathrm{TM}}$ (attL1/L2) sites. First, we deleted the Gateway ${ }^{\mathrm{TM}}$ attL2 site by digesting Tn7 TV1 with Sacl and Sacll, then bluntending and self-ligating the product to construct Tn7 TV1 ( $\triangle$ attL2; Supplementary Figure 4B). Next, we simultaneously deleted the attL1 site and inserted an expression cassette encoding the full-length Sf-Manl open reading frame ${ }^{9-11}$ followed by the AcMNPV p10 polyadenylation signal. This synthetic DNA was flanked by 5'Avrll/Pacl/Hpal and 3'-SnaBI/Nael sites and purchased from IDTDNA. The open reading frame included a silent mutation (T1800C) introduced to remove a Bsu36I recognition site. This synthetic DNA was then digested with Avrll and SnaBI and inserted into the Nhel and SnaBI sites of Tn7 TV1 ( $\Delta$ attL2), deleting the attL1 site in the process. An error-free clone of the resulting plasmid was designated Tn7 TV1-SfMan1 ( $\triangle$ attL1L2; Supplementary Figure 4C).

We then amplified a DNA fragment encoding the AcMNPV ie1 promoter preceded by the AcMNPV hr5 enhancer element using an in-house plasmid ${ }^{12}$ as the template. The resulting amplimer was digested with $\mathrm{Hpal}$ and $\mathrm{Pacl}$, cloned into the corresponding sites of Tn7 TV1-SfMan1 ( $\triangle$ attL1L2), and an error-free clone was designated Tn7 TV2 (Supplementary Figure 4D).

Finally, we amplified a DNA fragment encoding an AcMNPV 39K promoter-driven SfManll coding sequence with a silent mutation to delete a Bsu36I site (T2388C) followed by the AcMNPV pp31 polyadenylation signal. This fragment was produced using PaclSfManll-SP and Kpnl-SfManll-ASP primers with an in-house plasmid as the template. After digestion with $\mathrm{Kpnl}$ and $\mathrm{Pacl}$, this cassette was inserted into the same sites of $\mathrm{Tn} 7$ TV2, yielding Tn7 TV3 (Supplementary Figure 4E; Figure 2B). The annotated Tn7 TV3 sequence file is included in Supplementary Materials (Tn7 T3V.gb).

Construction of a parental modular Cre-LoxP donor vector 
To enable the insertion of genes into the LoxP site of PolyBac, we constructed a parental modular Cre-LoxP donor vector designed to accommodate up to 6 transgenes. First, we designed a sequence encoding an AcMNPV gp64 minimal 21/20 promoterdriven enhanced GFP (eGFP) marker, a chloramphenicol resistance marker, three distinct multiple cloning sites (Insert-L and $-R$, Multi-L and $-R$, and MCS-L and $-R$ ), a conditional $\mathrm{R} 6 \mathrm{~K} \gamma$ origin of replication, and a LoxP recombination site. We then purchased a plasmid carrying this sequence from DNA2.0 (Supplementary Figure 5A). This plasmid was used to insert an overlap PCR product encoding two back-to-back $39 \mathrm{~K}$ promoters (Ac39K and Op39K, derived from AcMNPV and Orgyia pseudotsugata $M N P V$, respectively) separated by the $D$. melanogaster white intron insulator ${ }^{13}$ (Wl; Supplementary Figure 5B). Finally, we digested the resulting plasmid with BstEll to remove the plasmid vector sequence and self-ligated the insert to create the parental modular Cre-LoxP donor vector (Supplementary Figure 5C). The annotated sequence file is included in Supplementary Materials (Parental Cre-LoxP donor vector.gb).

\section{Construction of Cre-LoxP donor vector derivatives}

We subsequently used the parental modular Cre-LoxP donor vector described in the preceding paragraph to construct a family of derivatives carrying various combinations of protein $\mathrm{N}$-glycosylation pathway genes (Figure 2C; Table 1). Each of these derivatives encoded MGAT1 and UGT. Additional protein $\mathrm{N}$-glycosylation pathway genes were sequentially inserted one or two at a time into this derivative to produce downstream derivatives encoding additional functions. This effort ultimately yielded a total of eight Cre-LoxP donor vector derivatives encoding up to six distinct protein $\mathrm{N}$ glycosylation pathway genes (Figure 2C). However, in the interests of brevity, we will focus the following description, which is supported by Supplementary Figure 6, on construction of the most extensively engineered donor vector derivative, UGG4 (Figure 2C), which encodes all six protein $\mathrm{N}$-glycosylation pathway genes. We used an analogous approach to construct the donor vector derivatives encoding smaller numbers of protein $N$-glycosylation pathway genes. Generally, the derivatives with even numbers of genes were produced by inserting expression cassettes encoding two genes at a time in back to back orientation, while the derivatives with odd numbers of genes were produced by inserting either one or two genes at a time, the latter as pairs in back to back orientation.

All cloning steps described in this section were performed using TransforMax ${ }^{\mathrm{TM}}$ EC100D ${ }^{\mathrm{TM}}$ pirt or pir-116 electro-competent E. coli strains (Lucigen Corporation, Middleton, $\mathrm{WI}$ ) and all transformants were selected with chloramphenicol.

Sequences encoding synthetic, codon-optimized MGAT1, -2, -4, -5, engineered B4GALT1, and UGT coding sequences were amplified using primers that introduced convenient restriction sites, with in-house plasmids as the templates ${ }^{14,}{ }^{15}$. The amplification products were digested with the appropriate restriction enzymes and inserted into complementary restriction sites in the left-hand (MCS-L) and/or right-hand (MCS-R) multiple cloning sites of the parental modular Cre-LoxP donor vector (Supplementary Figure $6 \mathrm{~A}$ ). In the case of UGG4, this produced three intermediate plasmids designated Cre-LoxP DV-1, -2 , and -3 , each encoding two distinct protein $N$ glycosylation pathway functions shown as arrows generically labeled A-F in 
Supplementary Figure 6 . We then excised the protein $\mathrm{N}$-glycosylation gene expression cassette from Cre-Lox DV-2 with Spel and inserted it into the Avrll site of Cre-Lox DV-1 to produce Cre-LoxP DV-1+2, which encodes four protein $N$-glycosylation pathway genes. Finally, we excised the protein $N$-glycosylation gene expression cassette from Cre-Lox DV-3 with Spel and inserted it into the Nhel site of Cre-Lox DV-1+2 to produce Cre-LoxP DV $-1+2+3$, which encodes six protein $\mathrm{N}$-glycosylation pathway genes in three back-to-back expression cassettes. An annotated Cre-LoxP DV-1+2+3 sequence file is included in Supplementary Materials (Cre-LoxP donor vector 123.gb).

\section{References - Supplementary materials and methods}

(1) Sharma, R. C.and Schimke, R. T. (1996) Preparation of electrocompetent E. coli using salt-free growth medium. Biotechniques 20, 42-44.

(2) Shizuya, H., Birren, B., Kim, U. J., Mancino, V., Slepak, T., Tachiiri, Y., and Simon, M. (1992) Cloning and stable maintenance of 300-kilobase-pair fragments of human DNA in Escherichia coli using an F-factor-based vector. Proc. Natl. Acad. Sci. U.S.A. 89, 8794-8797.

(3) Luckow, V. A., Lee, S. C., Barry, G. F., and Olins, P. O. (1993) Efficient generation of infectious recombinant baculoviruses by site-specific transposonmediated insertion of foreign genes into a baculovirus genome propagated in Escherichia coli. J. Virol. 67, 4566-4579.

(4) Maghodia, A. B., Jarvis, D. L., and Geisler, C. (2014) Complete Genome Sequence of the Autographa californica Multiple Nucleopolyhedrovirus Strain E2. Genome Announc. 2, e01202-01214.

(5) Maghodia, A. B., Geisler, C., and Jarvis, D. L. (2016) Characterization of an Sfrhabdovirus-negative Spodoptera frugiperda cell line as an alternative host for recombinant protein production in the baculovirus-insect cell system. Prot. Expr. Purif. 122, 45-55.

(6) Smith, G. E., Summers, M. D., and Fraser, M. J. (1983) Production of human beta interferon in insect cells infected with a baculovirus expression vector. Mol. Cell. Biol. 3, 2156-2165.

(7) O'Reilly, D. R., Miller, L. K., and Luckow, V. A. (1992) Baculovirus expression vectors, W.H. Freeman and Company, New York.

(8) Cornelissen, L. A. H. M., de Vries, R. P., de Boer-Luijtze, E. A., Rigter, A., Rottier, P. J. M., and de Haan, C. A. M. (2010) A single immunization with soluble recombinant trimeric hemagglutinin protects chickens against highly pathogenic avian influenza virus H5N1. Plos One 5, e10645.

(9) Kawar, Z., Romero, P. A., Herscovics, A., and Jarvis, D. L. (2000) N-glycan processing by a lepidopteran insect alpha1,2-mannosidase. Glycobiology 10, 347-355.

(10) Kawar, Z.and Jarvis, D. L. (2001) Biosynthesis and subcellular localization of a lepidopteran insect alpha 1,2-mannosidase. Insect Biochem. Mol. Biol. 31, 289297. 
(11) Kawar, Z., Herscovics, A., and Jarvis, D. L. (1997) Isolation and characterization of an alpha 1,2-mannosidase cDNA from the lepidopteran insect cell line Sf9. Glycobiology 7, 433-443.

(12) Jarvis, D. L., Weinkauf, C., and Guarino, L. A. (1996) Immediate-early baculovirus vectors for foreign gene expression in transformed or infected insect cells. Prot. Expr. Purif. 8, 191-203.

(13) Lee, Y. S.and Carthew, R. W. (2003) Making a better RNAi vector for Drosophila: use of intron spacers. Methods 30, 322-329.

(14) Geisler, C., Mabashi-Asazuma, H., Kuo, C. W., Khoo, K. H., and Jarvis, D. L. (2015) Engineering beta1,4-galactosyltransferase I to reduce secretion and enhance N-glycan elongation in insect cells. J. Biotechnol. 193, 52-65.

(15) Toth, A. M., Kuo, C. W., Khoo, K. H., and Jarvis, D. L. (2014) A new insect cell glycoengineering approach provides baculovirus-inducible glycogene expression and increases human-type glycosylation efficiency. J. Biotechnol. 182-183, 1929. 


\section{Supplemental Material Table 1: Primer sequences}

\begin{tabular}{|c|c|c|c|}
\hline Name & Sequence (5'-3') & $\begin{array}{l}5 \\
\text { Restriction } \\
\text { site }\end{array}$ & $\begin{array}{l}\text { Purpose I } \\
\text { Target }\end{array}$ \\
\hline Polh US SP & 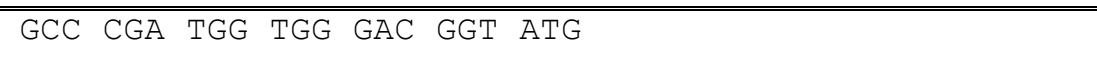 & EcoRV & Polyhedrin \\
\hline Polh US ASP & GTA ACG ATC GGG ACC GCT GGT GCG AGA AGC & Pvul & Upstream \\
\hline Polh DS SP & AAT AGG CCG GCC TTT GTG GTG GCC ATG ACG AAT CG & Fsel & Polyhedrin \\
\hline Polh DS ASP & ATA TAC GCG TAA ATT CTC CTT GAA GTT TCC CTG GTG & Mlul & Downstream \\
\hline Polh SP & ATG CCG GAT TAT TCA TAC CG & - & \multirow{2}{*}{$\begin{array}{l}\text { Polyhedrin } \\
\text { coding }\end{array}$} \\
\hline Polh ASP & TTA ATA CGC CGG ACC AGT G & - & \\
\hline HR2 SP & ACG ACT TCG AAG CGG AAT AAT TGC CAT ATG & Sphl & \multirow{2}{*}{ HR2 } \\
\hline HR2 ASP & GAG CTG CAT GCG TCA CGT GTA ATC AAT GTG & BstBI & \\
\hline EGT US SP & ATA GGA TAT CGC CAA ATC ATC ATG GAT TGC & EcoRV & \multirow{2}{*}{ EGT Upstream } \\
\hline EGT US ASP & CAT TAC CGG TGC CGC ATT TAC AGC AGT AAG & Agel & \\
\hline EGT DS SP & ATA GGC TAG CGC CAA TGT ACC GTA TTC AAA T & Nhel & \multirow{2}{*}{$\begin{array}{l}\text { EGT } \\
\text { Downstream }\end{array}$} \\
\hline EGT DS ASP & GAA TGG CCG GCC GAC ATA ACA AGC GCA GAC GG & Fsel & \\
\hline EGT SP & GTG TTT CCT ACG CCA GCT TAC & - & \multirow{2}{*}{ EGT coding } \\
\hline EGT ASP & GCG TTC TGT GAA TTT GAT GGC & - & \\
\hline HR5 SP & CAG CTA TGA CCA TGA TTA CG & - & \multirow{3}{*}{$\begin{array}{l}\text { Back-to-Back } \\
39 \mathrm{~K} \text { promoters }\end{array}$} \\
\hline $39 \mathrm{~K}$ ASP & GTT TGC TTC TTG TAA ACC TTT GA & - & \\
\hline $39 \mathrm{~K} \mathrm{SP}$ & $\begin{array}{l}\text { CGT AAT CAT GGT CAT AGC TGT ACA ATT CGC GCG CAC ATG } \\
\text { TTG }\end{array}$ & - & \\
\hline CC Zeo SP & $\begin{array}{lllllllll}\text { CCT ATT GAA GGA AGA ATC TTT AGA TGT GGG TTT GCT AAT } \\
\text { TTC GCC GCC ATG GAT CTC TGC AGC ACG TGT T }\end{array}$ & - & \multirow{2}{*}{$\begin{array}{l}\text { ChiA-vCath } \\
\text { flanking + Zeocin } \\
\text { coding }\end{array}$} \\
\hline CC Zeo ASP & $\begin{array}{lllllllll}\text { TTG TCT GCT TCG TAT GGA TAG TCG CTT TCC AGC TGT ACG } \\
\text { CCG CCC ATT TTA GAC ATG ATA AGA TAC ATT GAT GA }\end{array}$ & - & \\
\hline TAE25 SP1 & ATT AGG CGC GCC ATT TGT A & Ascl & \multirow{8}{*}{$\begin{array}{l}\text { Glycogene } \\
\text { coding }\end{array}$} \\
\hline TAE25 SP2 & ATT AGC GGC CGC ATT TGT A & Notl & \\
\hline UGT ASP & TCG TAA TGC CGG CTT AAG CCT TAG TGG GG & Nael & \\
\hline GNT-1 ASP & TCG TAA TGC CGG CTT AGT TCC AGG AGG GGT C & Nael & \\
\hline GNT-2 ASP & TCG TAA TGC CGG CTT ACT GCA GAC GAC GG & Nael & \\
\hline GNT-4 ASP & TCG TAA TGC CGG CTT AGT TGG TGG CCT TC & Nael & \\
\hline GNT-5 ASP & $\begin{array}{lllllll}\text { TCG TAA } & \text { TGC } & \text { CGG CTT ACA } & \text { GGC AGT } & \text { CCT } & \text { TG }\end{array}$ & Nael & \\
\hline B4GALT ASP & 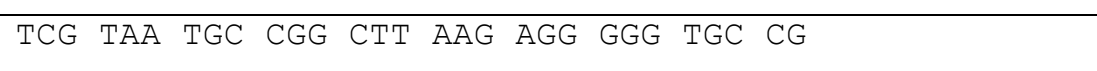 & Nael & \\
\hline GFP ASP1 & GGG ATC TTT AGA CAA TGC GC & - & \multirow{2}{*}{$\begin{array}{l}\text { UGT } \\
\text { Outside/lnside }\end{array}$} \\
\hline UGT Seq1 & GCG GAC AGT TCC TTA GAG TC & - & \\
\hline UGT Seq2 & GTG ATC CTG ATG ACC GGT G & - & \multirow{2}{*}{$\begin{array}{l}\text { UGT } \\
\text { Inside/Outside }\end{array}$} \\
\hline Cm ASP1 & GCT CAC CGT CTT TCA TTG CC & - & \\
\hline UGT SP & GAA ATA CGT GTC CCT GGG & - & \multirow{2}{*}{$\begin{array}{l}\text { UGT } \\
\text { coding }\end{array}$} \\
\hline UGT ASP & GAA GTC TTG CAG CCA GAA GT & - & \\
\hline
\end{tabular}




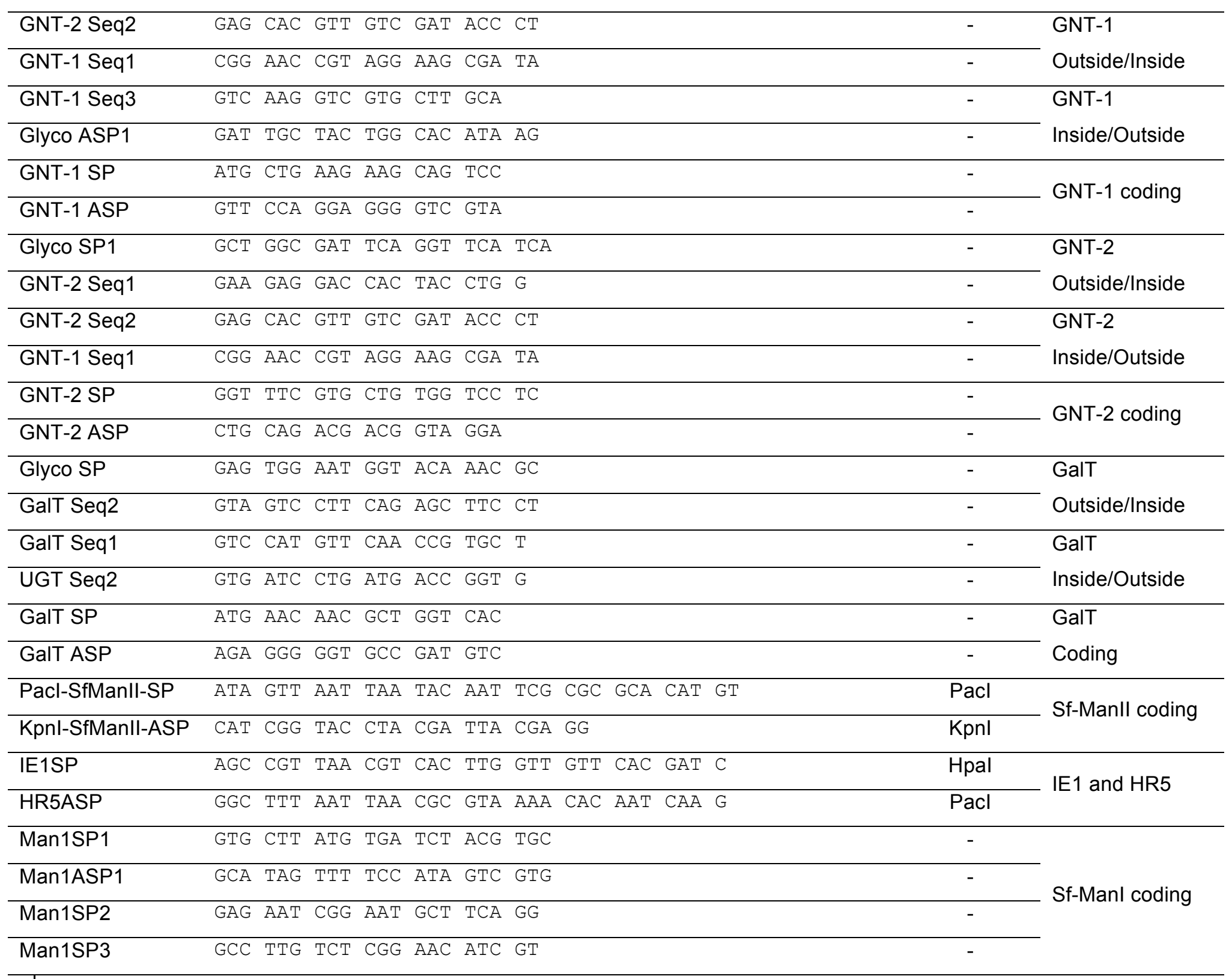




\section{Supplementary Figure Legends}

Figure S1. Construction of pPBTV-1. (A). A plasmid encoding a kanamycin resistance marker, mini-attTn7, and Lac Z alpha fragment flanked by multiple cloning sites (MCS-L, MCS-L) was purchased from DNA2.0 and an AcMNPV polyhedrin upstream (Polh US) sequence was amplified with a primer pair indicated by the left (sense) and right (antisense) arrows. The Polh US amplification product was then inserted into the synthetic DNA, as shown. (B). An AcMNPV polyhedrin downstream (Polh DS) sequence was amplified and inserted into the construct produced in $A$, as shown, to create pUC.LacZa-mini-attTn7.polh. (C). A mini-F replicon was excised from pBeloBac11 and inserted into pUC.LacZa-mini-attTn7.polh to create pUC.LacZa-mini-attTn7.mini-F.polh, as shown. (D). Finally, the AcMNPV HR2 enhancer element was amplified and inserted into pUC.LacZa-mini-attTn7.mini-F.polh to create pPBTV-1, as shown. The annotated pPBTV-1 sequence file is included in Supplementary Materials (pPBTV-1.gb).

Figure S2. Construction of pPBTV-2. (A). A plasmid encoding an AcMNPV p10promoter-driven LacZ-Rudolph Red Fluorescent Protein (RFP) fusion protein, an AcMNPV ie1(0)-promoter-driven herpes simplex virus type 2 thymidine kinase (TK) gene, Gateway ${ }^{\mathrm{TM}}$ (attR1/R2) recombination sites, a LoxP recombination site, and two multiple cloning sites (MCS $-L$ and $-R$ ), was purchased from DNA2.0. An AcMPNV egt upstream (EGT US) sequence was then amplified with a primer pair indicated by the left (sense) and right (antisense) arrows and inserted into the commercial parental plasmid, as shown. (B). Finally, an AcMNPV egt downstream (EGT DS) sequence was amplified and inserted into the daughter plasmid produced in A, as shown, to create pPBTV-2. The annotated pPBTV-2 sequence file is included in Supplementary Materials (pPBTV2.gb).

Figure S3. Genetic map of $\mathrm{Tn} 7$ helper plasmid. A plasmid encoding the $\operatorname{Tn} 7$ transposase genes tns $A$, tns $B$, tns $C$, and tns $D$ under the control of the $E$. coli LacZ promoter and an ampicillin resistance marker $\left(A m p^{R}\right)$ was designed and purchased from DNA2.0. The annotated Tn7 helper plasmid sequence file is included in Supplementary Materials ("Tn7 helper.gb").

Figure S4. Construction of Tn7 transfer vectors. (A). Tn7 TV1 is a plasmid encoding $\mathrm{Tn} 7$ right (Tn7R) and $\mathrm{Tn} 7$ left $(\mathrm{Tn} 7 \mathrm{~L})$ recognition sequences, Gateway recombination attL1 and attL2 sites, a gentamycin resistance marker $\left(\right.$ Gent $\left.{ }^{\mathrm{R}}\right)$, two multiple cloning sites (MCS)-L and -R, and the rH5 model glycoprotein used for this study under the control of the AcMNPV p6.9 promoter. This synthetic DNA sequence was designed, purchased from GeneArt, excised from the commercial vector to eliminate a kanamycin resistance marker, and subcloned into pUC19 to create Tn7 TV1. The annotated sequence file is included in Supplementary Materials (Tn7 TV1.gb). The Gateway ${ }^{\mathrm{TM}}$ att recombination sites were not required for this project. (B). Therefore, the attL2 site was deleted as shown to create Tn7 TV1 ( $\triangle$ attL2). (C). Next, the attL1 site was deleted and a synthetic Sf-Manl coding sequence (Kawar et al., 1997) was simultaneously inserted as shown to create Tn7 TV1-SfManl ( $\triangle$ attL1L2). (D). A DNA fragment encoding the AcMNPV ie1 promoter preceded by the AcMNPV hr5 enhancer element was amplified and inserted into Tn7 TV1-SfManl ( $\triangle$ attL1L2) as shown to create Tn7 TV2. (E). A DNA fragment encoding Sf-Manll under the control of the AcMNPV 39K promoter, followed by the AcMNPV pp31 polyadenylation signal was amplified and inserted into Tn7 TV2 to create 
Tn7 TV3. The annotated Tn7 TV3 sequence file is included in Supplementary Materials (Tn7 T3V.gb).

Figure S5. Construction of the parental modular Cre-LoxP donor vector. (A). A sequence encoding an enhanced GFP (eGFP) marker, chloramphenicol resistance marker $\left(C m p^{R}\right)$, three distinct multiple cloning sites (Insert- $L$ and $-R$, Multi-L and $-R$, and MCS-L and $-R$ ), conditional R6K $\gamma$ origin of replication, and LoxP recombination site was designed and a plasmid carrying this sequence was purchased from DNA2.0. (B). A PCR product encoding two back-to-back $39 \mathrm{~K}$ promoters (Ac39K and Op39K) separated by the drosophila white intron insulator (WI; Lee and Carthew, 2003) was inserted into this plasmid, as shown. (C). The daughter plasmid was digested to remove the vector sequence and the insert was self-ligated to create the parental modular Cre-LoxP donor vector product, as shown. The annotated parental modular Cre-LoxP donor vector sequence file is included in Supplementary Materials (Modular Cre-LoxP donor vector.gb).

Figure S6. Construction of modular Cre-LoxP donor vector derivatives. (A). The MCS-L and/or MCS-R sites in the parental modular Cre-LoxP donor vector were used to insert various transgenes encoding protein $\mathrm{N}$-glycosylation pathway functions. (B). The most complex modular Cre-LoxP donor vector derivative was constructed in a series of intermediate steps, which first involved inserting PCR amplimers encoding two distinct protein $\mathrm{N}$-glycosylation pathway genes in back-to-back orientation into the parental modular Cre-LoxP donor vector. Insertion of three cassettes encoding three distinct gene pairs generically marked A-F produced Cre-LoxP DV $-1,-2$, and -3 . Subsequently, an expression cassette encoding two of these genes was excised from Cre-LoxP DV-2 and inserted into Cre-LoxP DV-1 to produce Cre-LoxP DV-1+2, which has two expression cassettes encoding four protein $\mathrm{N}$-glycosylation pathway genes. Finally, an expression cassette encoding another pair of these genes was excised from Cre-LoxP DV-3 and inserted into Cre-LoxP DV-1+2 to produce Cre-LoxP DV-1+2+3, which has three expression cassettes encoding six back-to-back protein $\mathrm{N}$-glycosylation pathway genes. An annotated Cre-LoxP DV-1+2+3 sequence file is included in Supplementary Materials (Cre-LoxP donor vector 123.gb). 


\section{Supplementary Figure S1}

$A$ MCS-L $_{\text {Kan }}^{\text {Avrll,Pacl,Notl,Pmel }}$

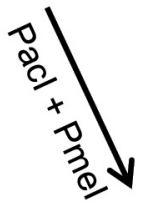

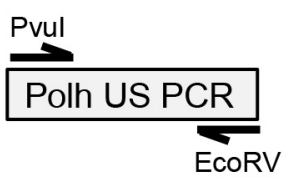

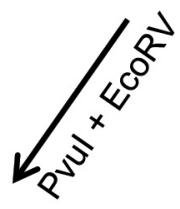

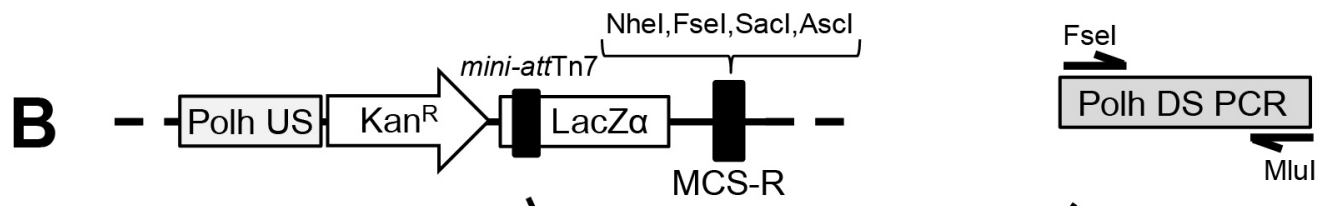
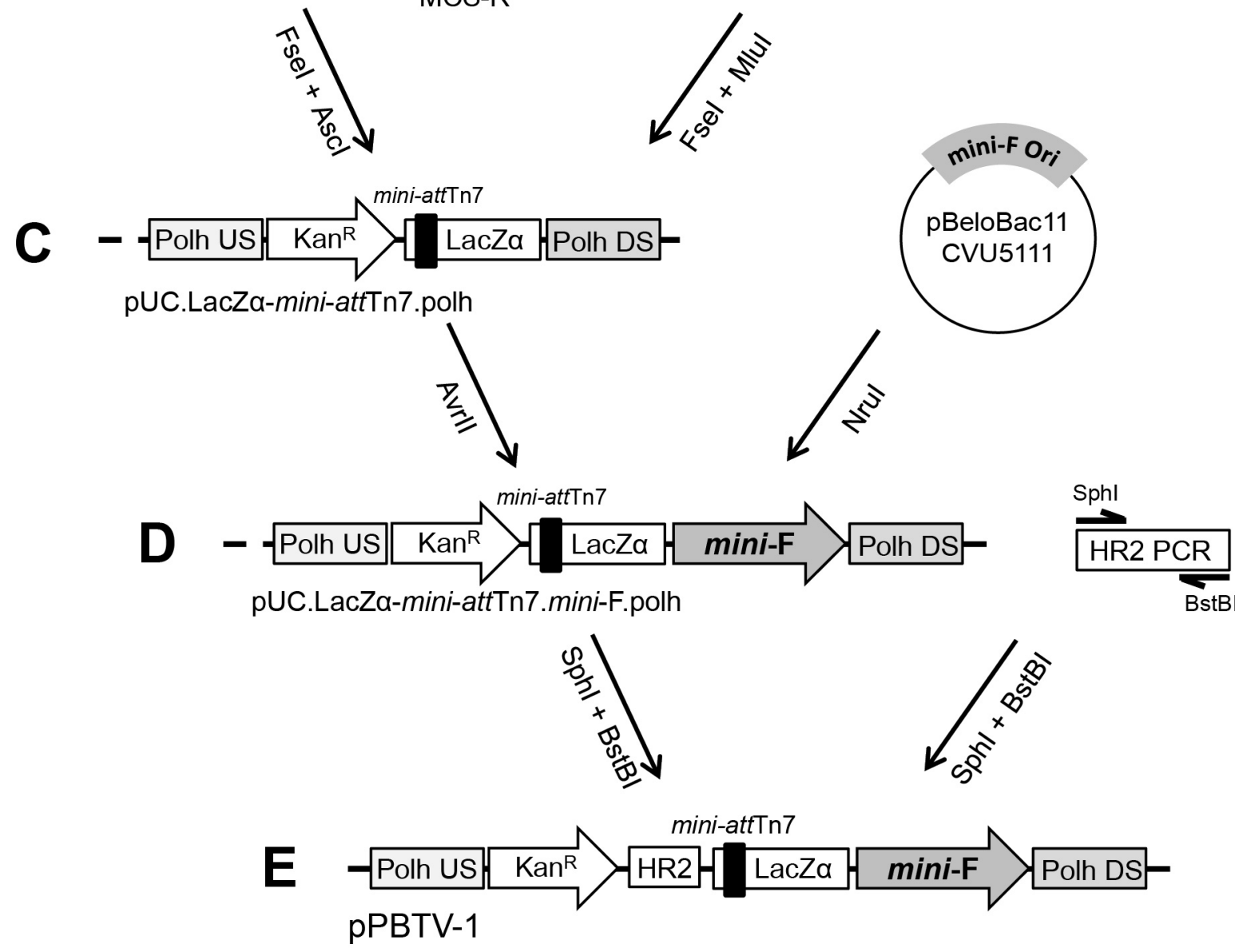
A

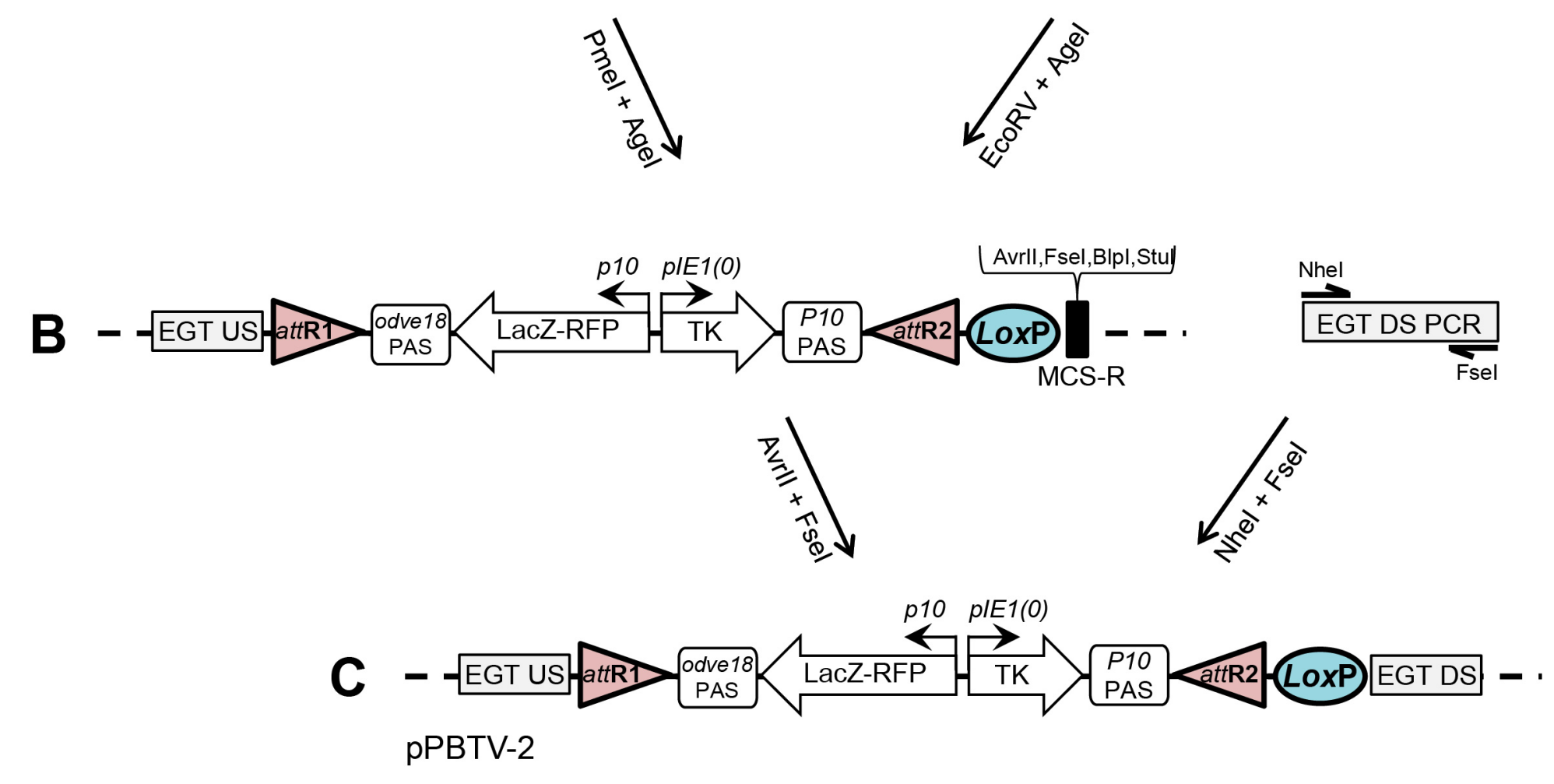




\section{Supplementary Figure S3}

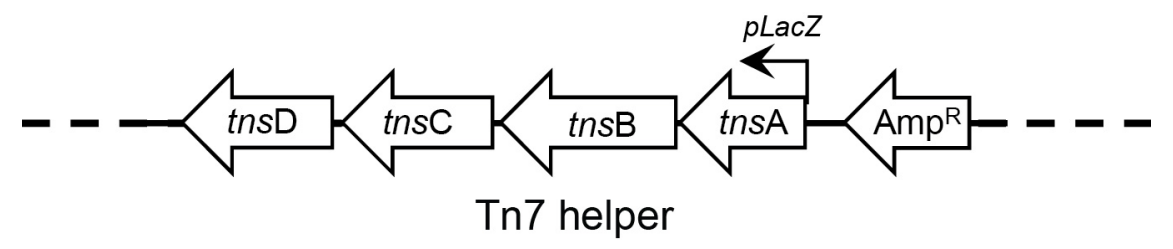


A

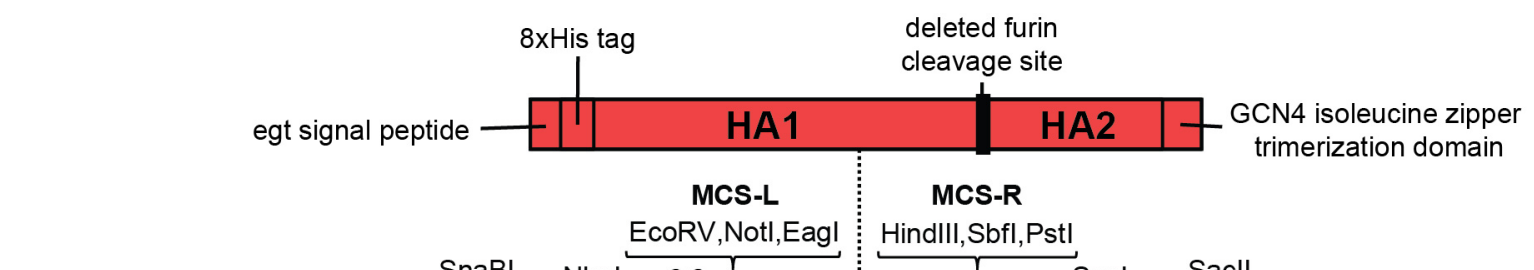

Sacl/Sacll,
Blunt, ligate

Tn7 TV1

B

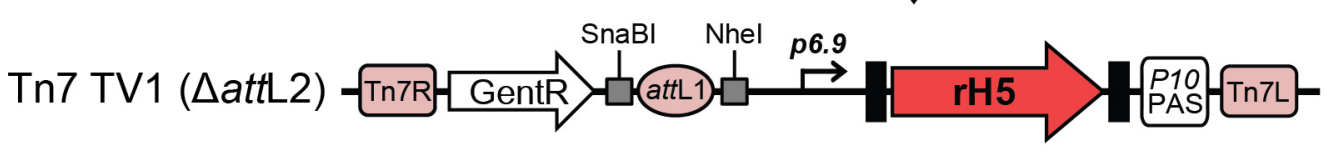

$\underbrace{\text { SnaBI, Nael Hpal, Pacl, Avrll }}$

B Tn7 TV1 $(\Delta$ attL2 $)$

$\downarrow$ SnaBI / Nhel

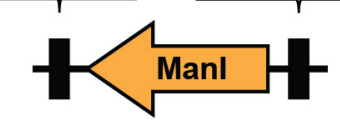

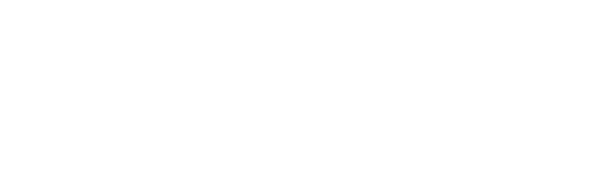
$\downarrow$

C Tn7 TV1-SfMan1 ( $\triangle$ attL1L2) -

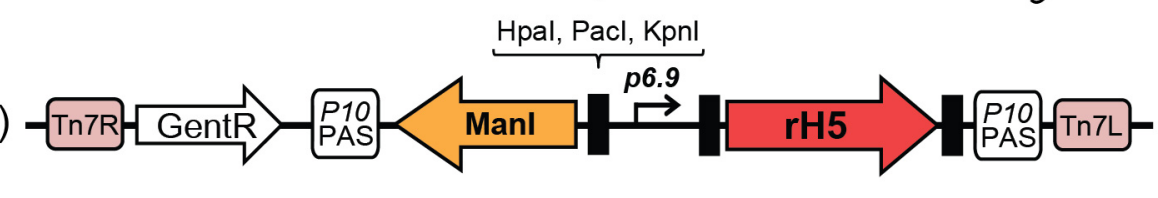

Hpal $\stackrel{\text { IIE1 Pacl }}{\longleftarrow}$

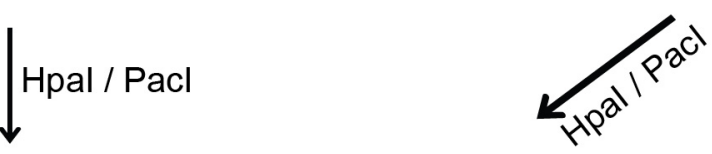

D

Tn7 TV2

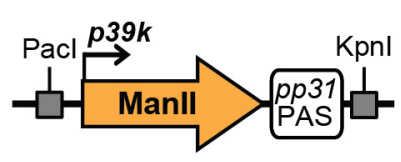

$\mathbf{E}$

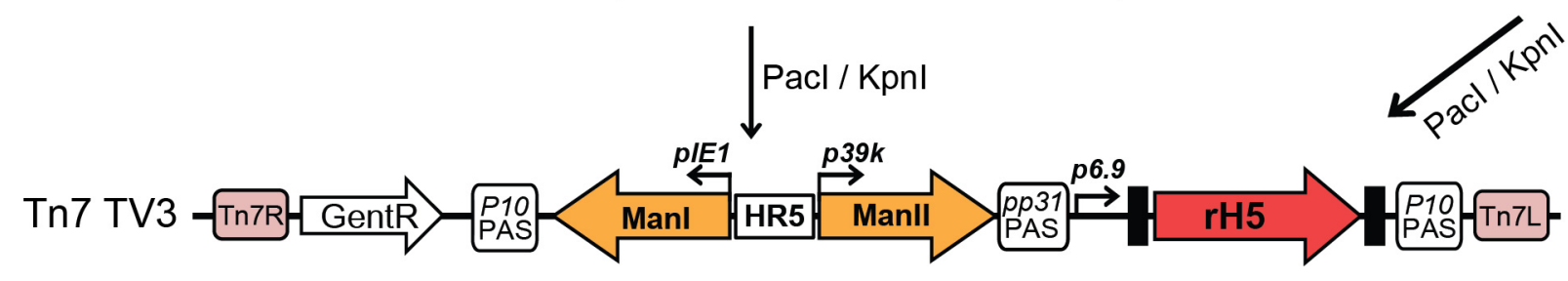




\section{Supplementary Figure S5}

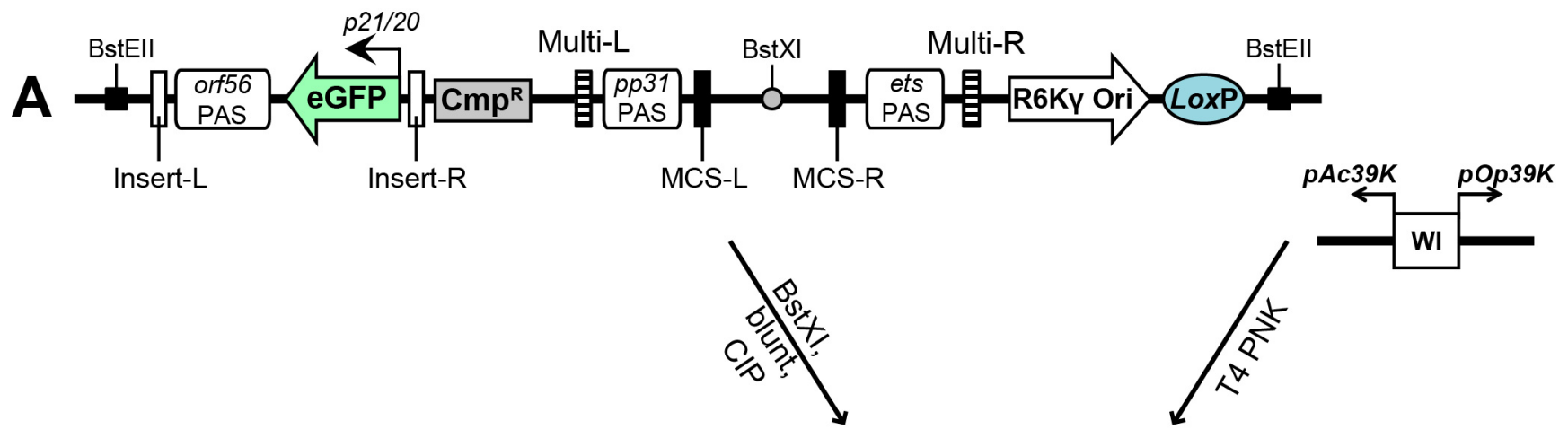

B
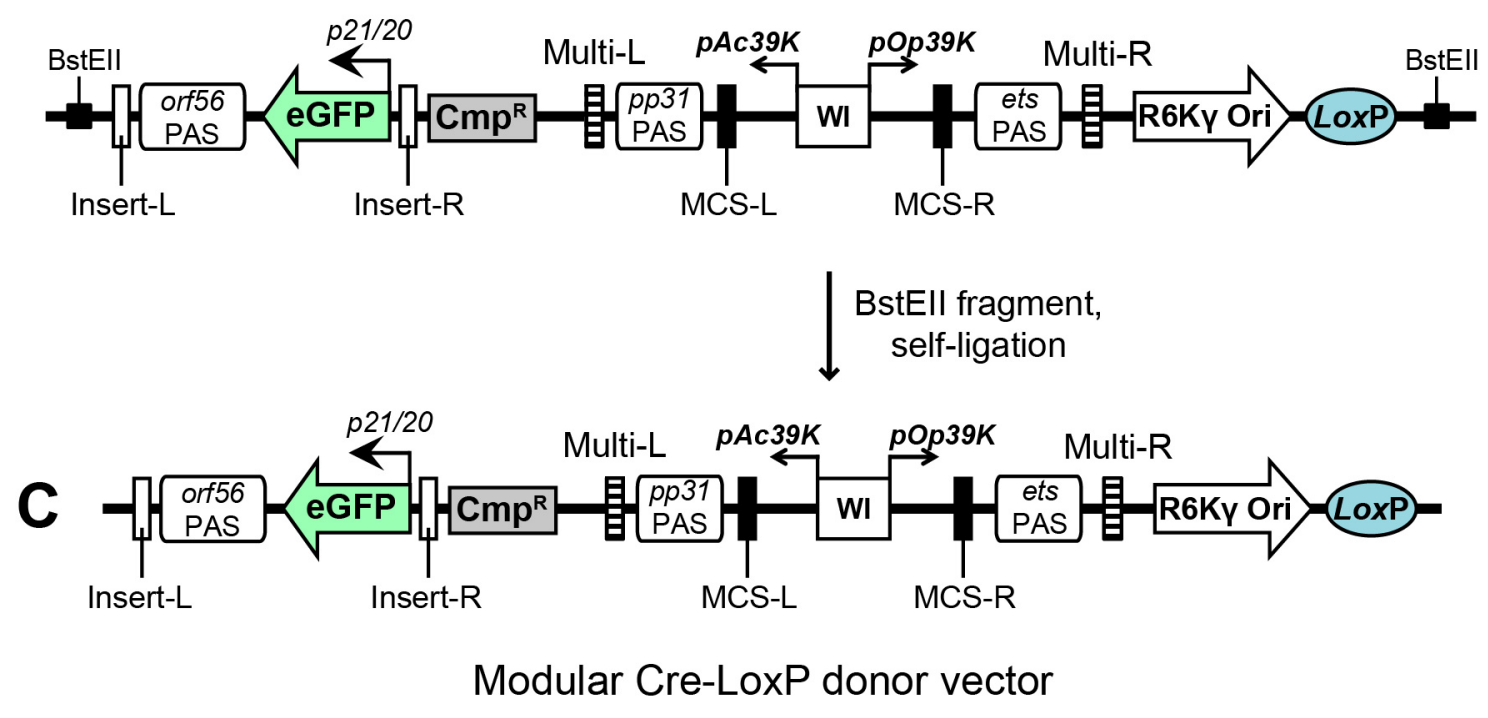


\section{Supplementary Figure S6}

$A$ Insert-L

B
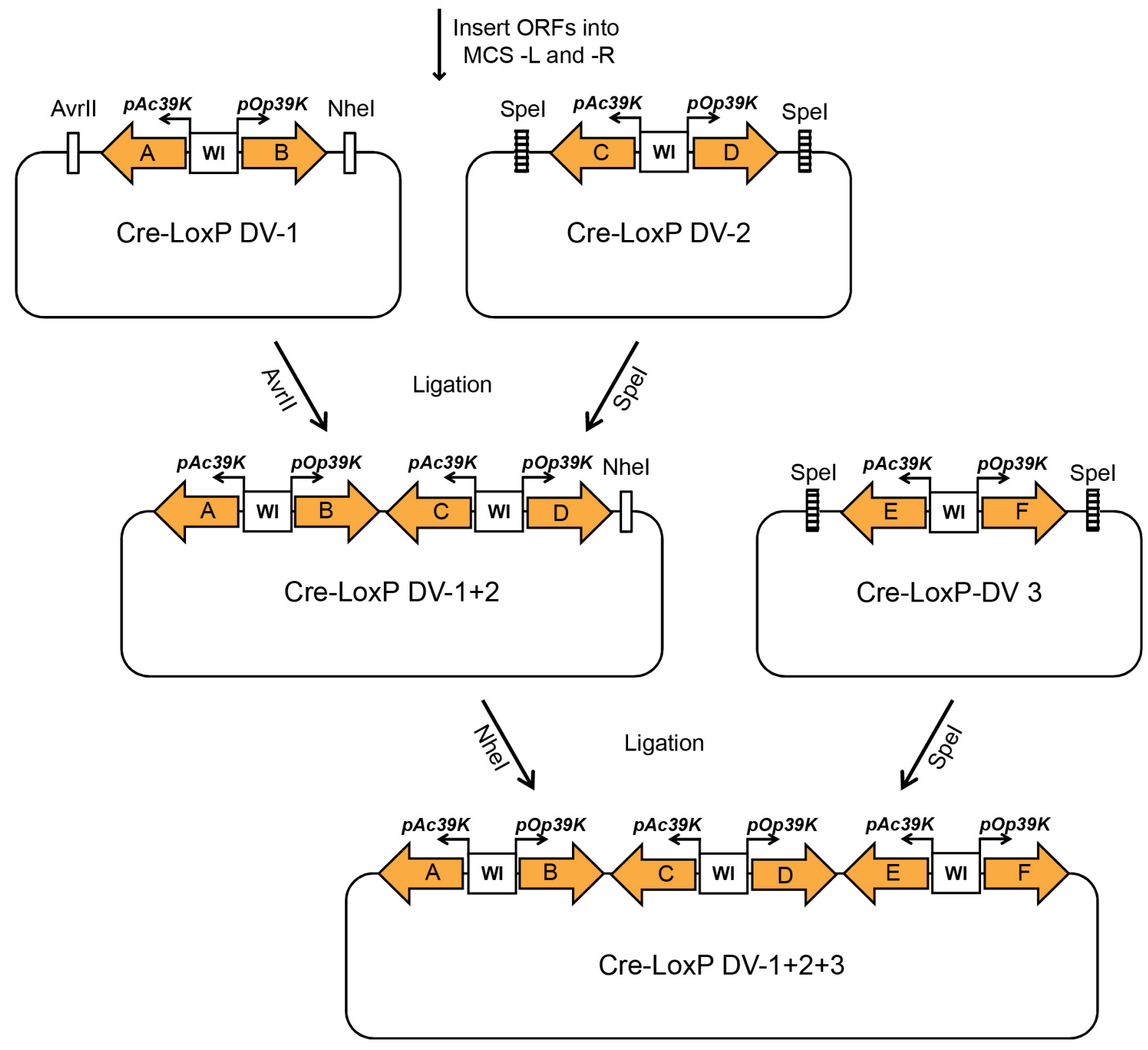